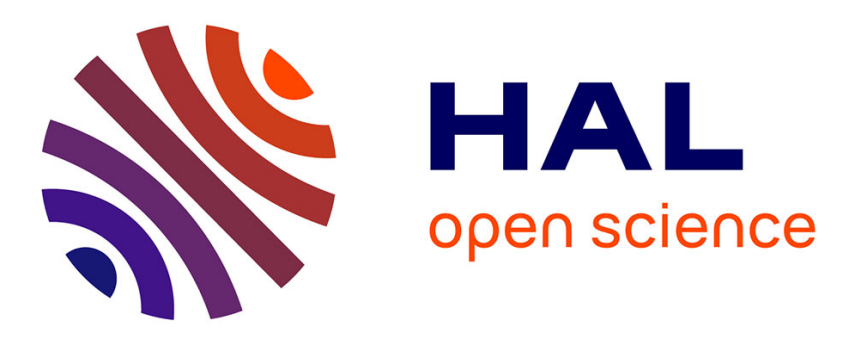

\title{
High resolution reflection tomographic diffractive microscopy
}

\author{
Merdan Sarmis, Bertrand Simon, Matthieu Debailleul, Bruno Colicchio, \\ Vincent Georges, Jean-Jacques Delaunay, Olivier Haeberle
}

\section{- To cite this version:}

Merdan Sarmis, Bertrand Simon, Matthieu Debailleul, Bruno Colicchio, Vincent Georges, et al.. High resolution reflection tomographic diffractive microscopy. Journal of Modern Optics, 2010, 57 (9), pp.740-745. 10.1080/09500341003624743 . hal-00827964

\section{HAL Id: hal-00827964 https://hal.science/hal-00827964}

Submitted on 2 Jul 2013

HAL is a multi-disciplinary open access archive for the deposit and dissemination of scientific research documents, whether they are published or not. The documents may come from teaching and research institutions in France or abroad, or from public or private research centers.
L'archive ouverte pluridisciplinaire HAL, est destinée au dépôt et à la diffusion de documents scientifiques de niveau recherche, publiés ou non, émanant des établissements d'enseignement et de recherche français ou étrangers, des laboratoires publics ou privés. 


\title{
MANUSCRIPT
}

\section{High resolution reflection tomographic diffractive microscopy}

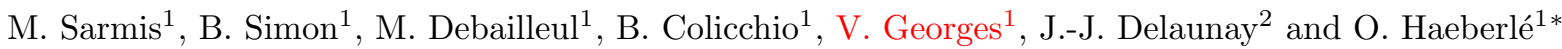 \\ ${ }^{1}$ Laboratoire MIPS-EA2332, Université de Haute-Alsace \\ IUT Mulhouse, 61 rue Albert Camus, 68093 Mulhouse Cedex, France \\ ${ }^{2}$ Department of Mechanical Engineering, School of Engineering, The University of Tokyo, 7-3-1 Hongo \\ Bunkyo-ku, Tokyo 113-8656, Japan
}

( )

\begin{abstract}
We have developped a tomographic diffractive microscope in reflection, using a high numerical aperture objective and equipped with a fluorescence confocal scanner. We describe the set-up and first images of a microscopic USAF target, obtained in holographic, diffractive tomographic, and confocal mode, and which reveal the higher resolution capabilities of this instrument. We also compare images obtained in transmission and in reflection, emphasizing the better optical sectionning capabilities of reflection diffractive tomographic microscopy.
\end{abstract}

Keywords: Tomographic Diffractive Microscopy; Fourier Optics; Holographic Interferometry; Resolution;

\section{Introduction}

Tomographic diffractive microscopy (TDM) $(1-7)$ is a wavefront-reconstruction based method (8) that allows for three-dimensional observations of microscopic objects. Using holographic techniques, the diffracted waves are recorded under various successive illumination directions. These informations are numerically recombined to reconstruct a three-dimensional image. In this technique, a single hologram represents in fact an object projection for a peculiar illumination angle. But in the spatial frequencies Fourier space, one hologram has a limited support : the transmitted band-pass is limited, which leads to a rather poor resolution. In order to increase the bandpass, an angular scanning has to be performed by using various successive illumination directions, allowing for the recording of higher frequencies (synthetic aperture process in Fourier space). Within this framework, holographic microscopy can be considered as a special case of tomographic diffractive microscopy, with only one single illumination angle.

As the object is reconstructed numerically, the physical model used for this numerical reconstruction determines its accuracy (9-11). For example, if using a filtered backprojection or inverse Radon transform (11-13), which does not take into account diffraction, the resolution is about the wavelength. Furthermore, such an approach is valid for slowly varying index of refraction objects only $(3,14)$. Reconstruction methods based on the Born $(6,7,11,15-17)$ or the Rytov (18) approximation take into account diffraction and lead to a better reconstruction quality $(10,11)$. Going even further, non-linear reconstruction schemes permit a resolution well beyond the Abbe limit in far-field (non-fluorescent) optical microscopy (19-21).

The tomographic diffractive microscopy technique can be used either in transmission $(6,7,15-$ 18), or in reflection (21-25). While transmission setups using high numerical aperture (NA) objectives have been described in the litterature by several authors $(7,13,15,16)$, leading in

*For correspondance. Email: olivier.haeberle@uha.fr 
some cases to a resolution in the $100 \mathrm{~nm}$ range (17), few experiments have been carried out in the reflection mode using a high NA objective (21).

In this paper, we describe the adaptation of the high-resolution transmission tomographic diffractive microscope we previously developped (15-17) (based on an Olympus IX71 inverted microscope body, equipped with an Olympus FV300 confocal scanner) into a high-resolution reflection tomographic diffractive microscope, and show results obtained when observing a microscopic USAF target.

\section{Principle of reflection tomographic diffractive microscopy}

We here only briefly recall the basic principle of tomographic diffractive microscopy, highlighting only the main differences existing between the transmission and the reflection modes.

In order to explain the synthetic aperture process, one first has to express the solution of the Helmoltz equation in the spatial frequencies Fourier space (see for example $(1,7,15)$ for a more complete explanation), which leads to:

$$
A\left(\mathbf{k}_{\mathbf{d}}\right) \propto \tilde{n}_{\delta}\left(\mathbf{k}_{\mathbf{d}}-\mathbf{k}_{\mathbf{i}}\right)
$$

In this equation, $A$ is the complex amplitude of the diffracted field in the Fourier space, $\tilde{n}_{\delta}$ the Fourier transform of the difference between the object refractive index $n(r)$ and the average index $n_{0}, \mathbf{k}_{d}$ a diffracted wavevector, $\mathbf{k}_{i}$ the illumination wave vector $\left(k_{d}=k_{i}=2 \pi / \lambda\right)$. For the sake of simplicity, we use here a simplified scalar model. A vectorial extension of this model has been proposed in ( 7$)$. The associated spatial frequencies of the scattered wave for a single illumination are thus located on the 2-D surface of the so-called Ewald sphere and interpreted as a Fourier transform of the sample relative index.

The synthetic aperture process in Fourier space is described by Figure 1. For clarity, Figures $1(\mathrm{a}-\mathrm{f})$ are 2-D representations in the $\left(k_{y}, k_{z}\right)$ plane, $k_{z}$ depicting the optical axis of the system. In Figures 1(a) and 1(d) one displays the incident wave (thick solid vector), the plain circle representing the extremities of the diffracted wavevectors (2-D cut through the Ewald sphere).

The set of Fourier components of the diffracted waves that can be collected is in fact limited by the numerical aperture of the microscope objective used in the detection system (dashed vectors). As a consequence, only a cap of the Ewald sphere can be recorded in practice. The set of Fourier components of the object (thin solid vectors) is then reconstructed by a translation of vector $-\mathbf{k}_{i}$. The main difference between transmission- and reflection diffractive tomography appears: in transmission, the set of reconstructed object frequencies depicts a cap of sphere, which summit is at the frequency origin (see Figure 1(a)), while in reflection, the reconstructed object frequencies cap of sphere is shifted toward higher frequencies (see Figure 1(d)).

When changing the angle of illumination, the set of detected diffracted waves is the same, but other object frequencies become recordable, thanks to the different translation to be done, as illustrated by Figure 1(b) and Figure 1(e). Note again that for the transmission construction, the detected cap of sphere passes through the frequency origin.

Finally, when a large number of incidences is used, one constructs an extended and filled support of object frequencies (Optical Transfer Function or OTF) ((see Figure 1(c), in grey), which exhibits in 2-D a characteristic so-called "butterfly" shape, centered on the origin, and characterized by a "missing-cone" of detected frequencies, which is at the origin of the low sectionning capabilities of transmission microscopes, including tomographic microscopes (17). We consider here the case where the numerical aperture at illumination is the same than the numerical aperture at detection, which explains the symmetric shape of the support with respect to the origin.

On the contrary, the set of recorded frequencies in reflection depicts a portion of a filled sphere, characterized by the same lateral extension than in transmission tomography, but now 
comprising high frequencies along the optical axis (see Figure 1(f)). From this construction, one therefore expects same transverse imaging capabilities in both configurations, but reflection microscopy should be characterized by superior sectionning capabilities along the optical axis. To the authors knowledge, this characteristic feature of reflection microscopy has not yet been demonstrated in the case of 3-D tomographic diffractive microscopy.

\section{Experimental set-up}

The setup is derived from our previous transmission tomographic microscope (15-17), which has been modified for reflection. We here briefly describes the new experimental set-up, highlighting the novelties with respect to our previous transmission system and forward the interested reader to previous publications $((7,13,15-17$, 22, 23), which give more technical details about the construction and use of a tomographic system.

Figure 2 describes our experiment. A HeNe laser $(\lambda=633 \mathrm{~nm})$ generates a coherent beam, which is divided into an illumination- and a reference wave. Optical fibers are used in order to simplify the transport of the illumination beam towards an oil immersion objective $(100 \mathrm{x}$, $\mathrm{NA}_{o b j}=1.4$ ) and toward the reference arm of the interferometer. The optical bench is adusted so that the illumination wave, injected into the objective using a recombination cube, exits this objective as a plane wave. The direction of the illumination is controlled using a tip-tilt mirror, so as to cover the full range of illumination angles for the objective.

Impiging onto the specimen, the illumination wave is diffracted. The objective collects the backward propagating diffracted wave and, associated to a Telan lens, refocuses an image onto the CCD detector. The recombination cube is used to mix the scattered beam with the reference beam, shaped as a plane wave, in order to record holograms. The phase and amplitude of the diffracted field is measured onto this hologram by reconstruction with a four-step phase shifting procedure, via the controlled piezo-electric mirror .

A numerical Fourier transform of the image then gives the data to be tiled onto the cap of the Ewald sphere corresponding to the illumination (see Fig. 1). The numerous caps of sphere are recombined in the Fourier space as described by Figures 1(a-c), and a final 3-D Fourier transform then permits to obtain a 3-D image of the observed sample. Throughout this work, we use this simplified reconstruction approach, which has proven to work very well for biological samples $(7,13,15-17)$, and apply it to a resolution test pattern. While strictly speaking not being valid for semiconductor or metallic specimens, the Born approximation has proven to work well for thin samples (22-25).

The microscope is also equipped with an Olympus FV300 confocal scanner working at $543 \mathrm{~nm}$ (HeNe green). Originally dedicated to fluorescence studies, this imaging system can also be used for reflection microscopy by removing the fluorescence filters, detecting then those illumination photons that are backscattered towards the objective. We use this confocal system to compare results obtained in holographic, tomographic and confocal imaging modes. In order to get the best images in terms of resolution, the pinhole was closed at minimum, and 10 images are recorded and averaged for each plane, in order to improve the signal to noise ratio.

\section{Experimental results}

In order to evaluate the imaging capabilities of our system, we have imaged a microscopic 1951 USAF resolution test chart. It was obtained by etching a $75 \mathrm{~nm}$ thickness platinum layer, sputtered onto a Matsunami Glass slide $(18 \times 18 \times 0.15 \mathrm{~mm})$. The USAF chart was produced using focused ion beam (FIB) etching technique. A high quality pattern can be etched, which can be simultaneously controlled using FIB imaging. The largest line is $2.5 \mu \mathrm{m}$ long and $0.5 \mu \mathrm{m}$ large and six of these elements are used to form a subpattern (made out of two structures of three equally spaced elements). The whole test pattern comprises three groups (numbered 1 to 3 ) 
of six subpatterns (numbered 1 to 6 ), the dimensions of the subpatterns following a geometric progression of factor $2^{-1 / 6}$. With this kind of test chart, it is common to express the resolution in terms of line pairs per micrometer $(\mathrm{lp} / \mu \mathrm{m})$. The resolving power of the system can then be estimated by identifying the smallest visible pattern, and is in that case given by the equation:

$$
R(l p / \mu m)=2^{(\text {Group }-1+((\text { Element-1)/6) })}
$$

We checked first that the pattern was correctly etched by analysing the FIB image. We could clearly identify element 2 of group 3, a structure made out of bars $111 \mathrm{~nm}$ wide and $555 \mathrm{~nm}$ long, or $4.5 \mathrm{lp} / \mu \mathrm{m}$. The four smaller elements of group 3 were not satisfyingly etched.

Figure 3 shows the results we obtained in (a) holographic, (b) confocal and (c) tomographic imaging modes. Note that the contrast is inverted between holographic or tomographic images and the confocal one, but this characteristic has no influence on the resolution estimation. The quality of the images clearly improves a lot when switching from holography to tomography. The confocal image is characterized by a very good contrast and low noise, which is easily explained by the nature of the sample, which is essentially a mirror, and is between holography and tomography in terms of resolution. The signal to noise ratio also improves from holography to tomography, thanks to the high redundancy between the numerous sequential acquisitions in tomography (16).

When analysing these images, one concludes that the smallest structure identified without ambiguity on the holographic image is subpattern 3 of group 1. On the confocal image subpattern 2 of group 2 can be clearly identified (for subpattern 3 of group 2, the horizontal bars are distinguishable, but the vertical ones are merged, which leads us not to consider this structure has being resolved) and on the tomographic image subpattern 3 of group 2 can still but hardly be recognized. These convert into a resolution of $1.26 \mathrm{lp} / \mu \mathrm{m}$ for holographic-, $2.24 \mathrm{lp} / \mu \mathrm{m}$ for confocal-, and $2.52 \mathrm{lp} / \mu \mathrm{m}$ for tomographic microscopy, respectively. One notes that the theoretical factor two of resolution improvement between holographic microscopy and tomographic microscopy is obtained (7). However, the 11 percent improvement in resolution between confocal microscopy and tomographic microscopy is much smaller than what we obtained when comparing transmission tomographic microscopy and fluorescence microscopy $(130 \mathrm{~nm}$ versus $200 \mathrm{~nm}$, or 35 percent (17)). It has to be recalled that, in terms of resolution, the tomographic microscope is in fact handicaped because working at a slightly larger wavelength that the confocal system (633 $\mathrm{nm}$ versus $543 \mathrm{~nm})$.

In theory, a confocal microscope has a resolution about 40 percent better than a conventional microscope, but that assumes an infinitely small pinhole. In practice, the confocal lateral resolution is only marginally better, because of the finite-size pinhole (usually with a diameter of one Airy disk), which has to be used for sufficient photon detection (the main interest of confocal microscopy is its far superior optical sectioning capabilities). Estimated from the dimensions of the captured frequency support, and taking into account coherent illumination and reconstruction of the data $(7,26)$, a tomographic diffractive microscope should have a twice better resolution than a wide-field microscope working at same numerical aperture with incoherent light at same wavelength.

This would translate into a lateral resolution of about $190 \mathrm{~nm}$ at $\lambda=542 \mathrm{~nm}$ and NA=1.4 for the confocal system (223 nm measured), $113 \mathrm{~nm}$ at $\lambda=633 \mathrm{~nm}$ for the tomographic setup (198 nm measured). The large difference between theoretical and experimental resolution for tomographic microscopy, the smaller-than-expected difference between confocal and tomographic resolution, and the fact that the horizontal and vertical structures exhibit slight differences, probably because of polarization effects, which are not taken into account in the reconstruction model (see for example subpattern 1 of group 3 in tomography), all suggest the limitations of our approach based on the to the Born approximation that is not perfectly adapted for this kind of samples.

Finally, we compared the 3-D optical sectioning performances of transmission tomographic 
diffractive microscopy, reflection confocal microscopy and reflection tomographic diffractive microscopy. Figure 4 shows (x-z)views of 3-D stacks obtained with the three methods, cut along the line depicted by the arrows in Figures $3(\mathrm{~b}, \mathrm{c})$. The image of the test pattern in the $(\mathrm{x}-\mathrm{y})$ focal plane obtained with transmission tomographic diffractive microscopy is almost identical to the reflection tomographic diffractive image and is therefore not shown here. Figure 4(a) emphasizes the rather low optical sectioning capability of 3-D transmission tomographic diffractive microscopy (17). As explained previously, transmission microscopes are characterized by a socalled missing cone of non-transmitted frequencies along the optical axis (see Figure 1(c)), which translates into poor imaging of those interfaces, which are perpendicular to the optical axis. One can see the effects of this missing cone as large vertical artefacts in the object reconstruction.

On the contrary, a confocal microscope has no missing cone, and provides much better sectioning, as illustrated by Figure 4(b). Figure 4(c) shows the image obtained with reflection tomographic diffractive microscopy. It demonstrates the much better optical sectioning properties of this setup, as deduced from the shape and location of its Optical Transfer Function (see Figure 1(f)), compared to that of transmission tomographic diffractive microscopy (see Figure 1(c)). Reflection tomographic diffractive microscopy therefore provides a higher lateral resolution (than holographic microscopy) with the high optical sectioning ability of confocal reflection microscopy.

Note again that the simplified reconstruction approach we use, based on the first Born approximation, gives limited results in terms of reconstruction precision. One can for example notice rebounds visible along the optical axis, near the surface of the object (see Figure 4(c)). Such artifacts are also visible in the $(\mathrm{x}-\mathrm{y})$ plane on high resolution transmission tomographic diffractive microscopy images (17). These rebounds testify to the image reconstruction process, which is performed in Fourier space, and can be interpreted in terms of Gibbs oscillations. Furthermore, contrary to transmission tomographic diffractive microscopy, the OTF of reflection tomographic diffractive microscopy is not symmetric with respect to the frequency origin, and misses the low frequencies. It may therefore be viewed as a high pass filter, which enhances transitions. More elaborate reconstruction procedure should give more accurate reconstructions (20, 21).

These images however show that, even taking into account the limitations of our reconstruction technique, reflection tomographic diffractive microscopy shall be of high interest for surfaces and interfaces studies, such as for example in surface physics, or for semiconductor inspection not only because longer working distance systems may be used (22-25), but also because of the much higher intrinsic lateral resolution and optical sectioning it permits.

\section{Conclusion}

We have developed a tomographic diffractive microscope in reflection, using a high numerical aperture objective. The numerical reconstruction of the specimen image being based on the first Born approximation, our setup is limited when observing metallic structures, which are known to be out of the strict domain of validity of this approach. Note also that the test we used in this preliminary work yields a precise value for the resolution, but is semi-quantitative only, as it depends on the user's definition of acceptable blur.

However, despite these limitations, using a microscopic 1951 USAF test pattern, we could show that the gain in resolution, compared to holography was as predicted by theory. The resolution of the tomographic system also compares favourably with reflection confocal microscopy. In this case also, a modest but noticeable gain in resolution has been observed, in favour of diffractive tomography.

We also compared the 3-D optical sectioning capabilities of transmission tomographic diffractive microscopy, reflection confocal microscopy and reflection tomographic diffractive microscopy. The former is characterized by rather low optical sectioning properties, explained by the so-called missing cone characteristic of all transmission microscopes. Reflection confocal microscopy provides much better optical sectioning. 
Reflection tomographic diffractive microscopy has the advantage of combining the higher lateral resolution of tomographic systems with the good optical sectioning allowed by a reflection setup.

\section{Acknowledgements}

The authors would like to thank H. Giovaninni, E. Maire and A. Sentenac for helpful discussions about tomographic diffractive microscopy in reflection, and the anonymous referee for her/his helpful comments.

\section{References}

(1) E. Wolf, Three-dimensional structure determination of semi transparent objects from holographic data, Opt. Comm. 1, 153-156 (1969).

(2) R. Dandliker and K. Weiss, Reconstruction of three-dimensional refractive index from scattered waves, Opt. Comm. 1, 323-328 (1970).

(3) W. H. Carter and P.-C. Ho, Reconstruction of inhomogeneous scattering objects from holograms, App. Opt. 13, 162-172 (1974).

(4) A.F. Fercher, H. Bartelt, H. Becker, and E. Wiltschko, Image formation by inversion of scattered field data : experiments and computational simulations, App. Opt. 18, 2427-2439 (1979).

(5) M. H. Malecki, A. J. Devaney, and A. Schatzberg, Tomographic reconstruction from optical scaterred intensities, J. Opt. Soc. Am. A 9, 1356-1363 (1992).

(6) T. Noda, S. Kawata, and S. Minami, Three-dimensional phase-contrast imaging by a computed-tomography microscope Applied Optics 31 670-674 (1992)

(7) V. Lauer, New approach to optical diffraction tomography yielding a vector equation of diffraction tomography and a novel tomographic microscope, J. Microscopy 205, 165-176 (2002).

(8) D. Gabor, A new microscopic principle, Nature 161, 777-778 (1948).

(9) A. C. Kak and M. Slaney, Principles of Computerized Tomographic Imaging (IEEE Press, 1988).

(10) W. Gorski and W. Osten, Tomographic imaging of photonic crystal fibers, Opt. Lett. 32, 1977-1979 (2007).

(11) S. Vertu, I. Yamada, J.-J. Delaunay and O. Haeberlé 2008 Tomographic observation of transparent objects under coherent illumination and reconstruction by filtered backprojection and Fourier diffraction theorem SPIE Proceedings $\mathbf{6 8 6 1} 686103$

(12) F. Charrière, A. Marian, F. Montfort, J. Kuehn, T. Colomb, E. Cuche, P. Marquet, and C. Depeursinge, Cell refractive index tomography by digital holographic microscopy, Opt. Lett. 31, 178-180 (2006).

(13) W. Choi, C. Fang-Yen, K. Badizadegan, S. Oh, N. Lue, R. R. Dasari, and M. S. Feld, Tomographic phase microscopy, Nature Methods 4, 717-719 (2007).

(14) M. Slaney, A. C. Kak, and L. E. Larsen, Limitations of Imaging with First-Order Diffraction Tomography, IEEE Trans. Microwave Theory Tech. MTT-32, 860-874 (1984).

(15) M. Debailleul, B. Simon, V. Georges, O. Haeberlé, and V. Lauer, Holographic microscopy and diffractive microtomography of transparent samples, Meas. Sci. Technol. 19, 074009 (2008).

(16) B. Simon, M. Debailleul, V. Georges, V. Lauer, and O. Haeberlé, Tomographic diffractive microscopy of transparent samples, Eur. Phys. J. App. Phys. 44, 29-35 (2008).

(17) M. Debailleul, V. Georges, B. Simon, R. Morin and O. Haeberlé, High-resolution three-dimensional tomographic diffractive microscopy of transparent inorganic and biological samples, Opt. Lett. 34, 79-81 (2009)

(18) Y. Sung, W. Choi, C. Fang-Yen, K. Badizadegan, R. R. Dasari, and M. S. Feld, Optical diffraction tomography for high resolution live cell imaging, Opt. Exp. 17, 266-277 (2009)

(19) A. Sentenac, P. Chaumet, and K. Belkebir, Beyond the Rayleigh Criterion: Grating Assisted Far-Field Optical Diffraction Tomography, Phys. Rev. Lett. 97, 243901 (2006)

(20) Kamal Belkebir, Patrick C. Chaumet, and Anne Sentenac, Influence of multiple scattering on three-dimensional imaging with optical diffraction tomography, J. Opt. Soc. Am. A 23, 586-595 (2006)

(21) G. Maire, F. Drsek, J. Girard, H. Giovannini, A. Talneau, D. Konan, K. Belkebir, P. C. Chaumet, and A. Sentenac, Experimental Demonstration of Quantitative Imaging beyond Abbes Limit with Optical Diffraction Tomography, Phys. Rev. Lett. 102, 213905 (2009)

(22) S. A. Alexandrov, T. R. Hillman, T. Gutzler, and D. D. Sampson, Synthetic Aperture Fourier Holographic Optical Microscopy, Phys. Rev. Let. 97, 168102 (2006)

(23) V. Mico, Z. Zalevsky, P. García-Martínez and J. García, Synthetic aperture superresolution with multiple off-axis holograms, J. Opt. Soc. Am. A 23, 3162-3170 (2006)

(24) V. Mico, Z. Zalevsky, and J. García, Synthetic aperture microscopy using off-axis illumination and polarization coding, Opt. Comm. 276, 209-217 (2007)

(25) L. Martínez-León and B. Javidi, Synthetic aperture single-exposure on-axis digital holography, Opt. Exp. 16, 161-169 (2008)

(26) O. Haeberlé, A. Sentenac and H. Giovaninni, An introduction to diffractive tomographic microscopy, in Modern Research and Educational Topics in Microscopy (A. Méndez-Vilas and J. Díaz (Eds.) Formatex, 2007). 

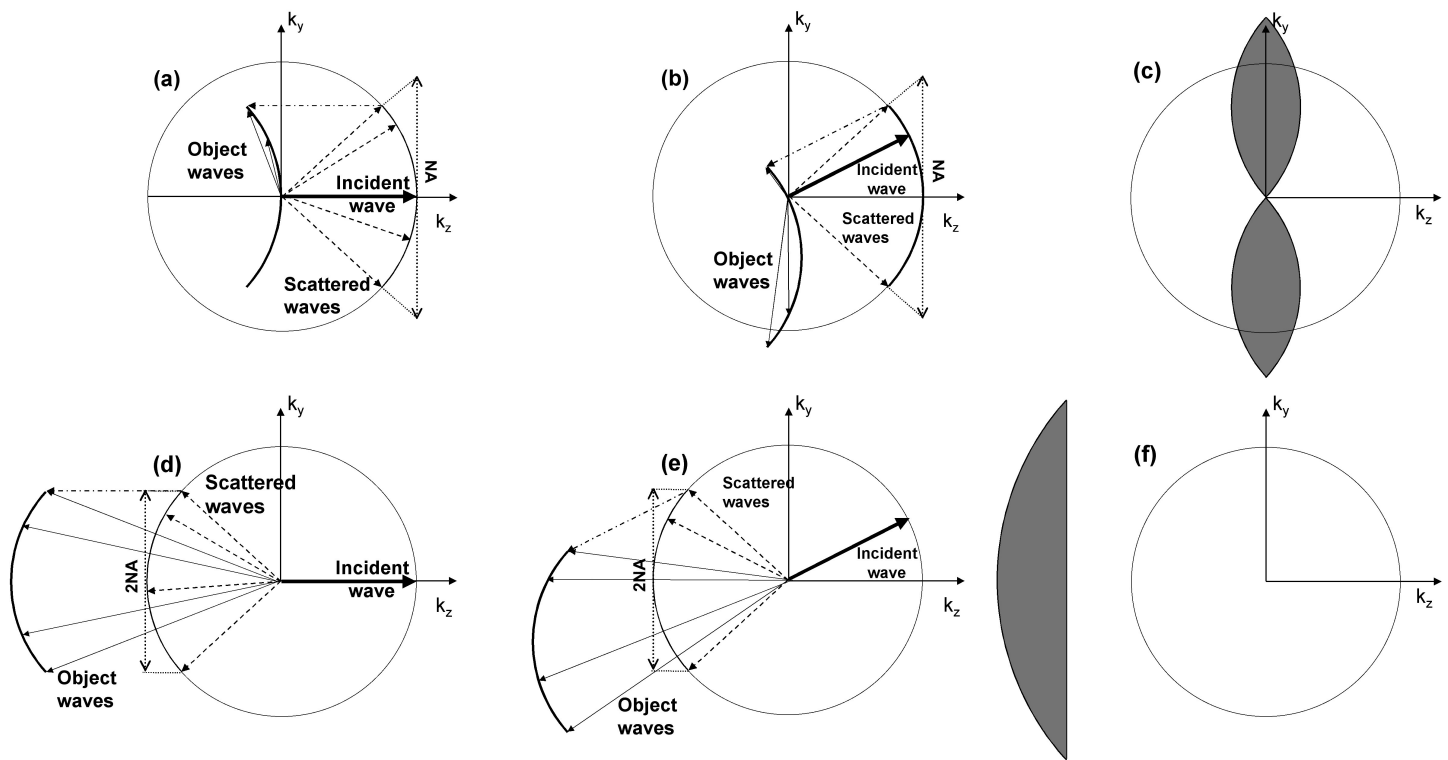

Figure 1. Principle of tomographic diffractive microscopy: synthetic aperture process in Fourier space for (a-c): transmission and (d-f): reflection modes. $(\mathrm{a}, \mathrm{d})$ : for normal incidence. (b,e): for inclined incidence. (c,f): final frequency supports (in grey) for a large number of illumination angles. Note the drastically different frequency support shapes for both configurations. 


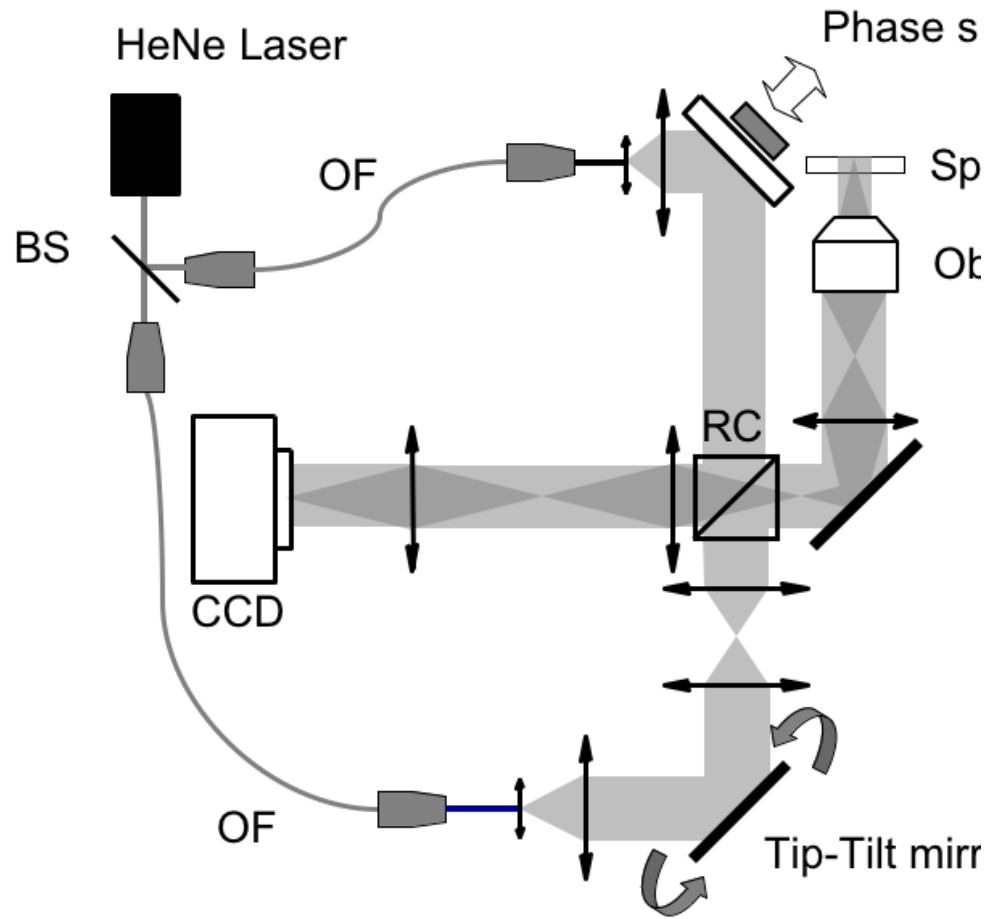

Figure 2. Sketch of our tomographic microscope in reflection (BS: beam splitter, OF: optical fiber, RC: recombination cube). Scanning confocal microscopy has not been representated. 

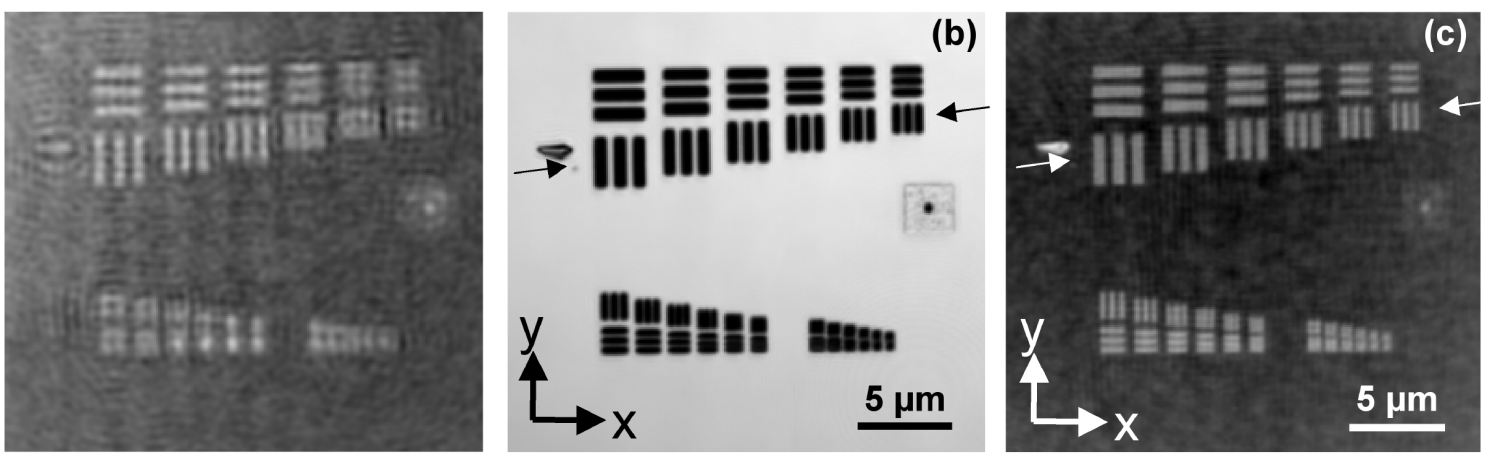

Figure 3. Images of a microscopic USAF target in (a): holographic reflection microscopy, (b) reflection confocal microscopy, and (c) reflection tomographic diffractive microscopy 

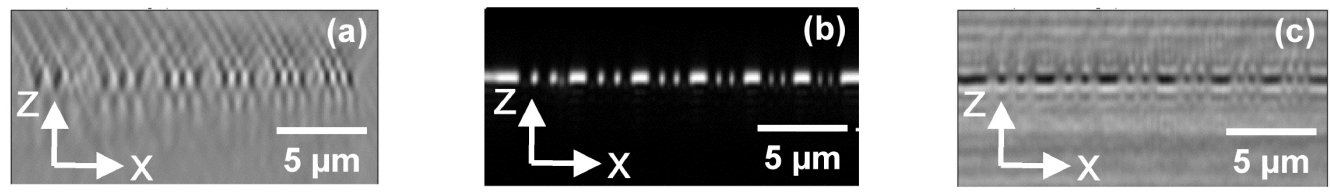

Figure 4. Images of a microscopic USAF target along the optical axis in (a): transmission tomographic diffractive microscopy, (b) reflection confocal microscopy, and (c) reflection tomographic diffractive microscopy 